\title{
The Spectrum and the Stability of the Chebyshev Collocation Operator for Transonic Flow
}

\author{
By Dalia Fishelov
}

\begin{abstract}
The extension of spectral methods to the small disturbance equation of transonic flow is considered. It is shown that the real parts of the eigenvalues of its spatial operator are nonpositive. Two schemes are considered; the first is spectral in the $x$ and $y$ variables, while the second is spectral in $x$ and of second order in $y$. Stability for the second scheme is proved. Similar results hold for the two-dimensional heat equation.
\end{abstract}

1. Introduction. Consider the transonic small disturbance equation

$$
2 \phi_{t x}=\left(k \phi_{x}-\frac{\gamma+1}{2} \phi_{x}^{2}\right)_{x}+4 \phi_{y y}
$$

and its steady state version

$$
\left(k \phi_{x}-\frac{\gamma+1}{2} \phi_{x}^{2}\right)_{x}+4 \phi_{y y}=0,
$$

where $\phi$ is the velocity potential, $k$ and $\gamma$ are positive constants. These equations model subsonic and supersonic flow close to the local speed of sound. The flow is assumed to be that of an inviscid perfect gas.

The time-dependent equation (1.1) and the time-independent equation (1.2) are of practical importance in computing flows around an airplane flying at a speed close to Mach 1. They are also used as models for more complex problems, since they describe important phenomena such as shock waves and discontinuities of partial derivatives of the solution $\phi$ near the tips of the airplane. They constitute a good model because their steady state (1.2) is of mixed type, which is easily seen from the following form of (1.2):

$$
\left(k-(\gamma+1) \phi_{x}\right) \phi_{x x}+4 \phi_{y y}=0
$$

(1.3) is elliptic for $\phi_{x}<k /(\gamma+1)$ and hyperbolic for $\phi_{x}>k /(\gamma+1)$. In addition, the time-dependent equation is a model for other problems in two space variables approximated by spectral methods, for which we examine stability and convergence to a steady state solution.

The purpose of this paper is to give theoretical support to the schemes, presented here and in [6], for solving the small disturbance equation, using Chebyshev spectral methods. Numerical results are given in [6] and [5]. The present paper contains

Received February 3, 1987; revised January 18, 1988

1980 Mathematics Subject Classification (1985 Revision). Primary 76H05, 33A65, 35P15.

Key words and phrases. Spectral methods, transonic flow, stability, Chebyshev polynomials, eigenvalue problems. 
eight sections. In Section 2 we present the small disturbance equation (SDE) of transonic flow and review finite difference methods [13], [4] for solving it numerically. Section 3 presents a proof that the linearized differential problem is well posed.

In Section 4 we present two schemes for solving the SDE. Both schemes are spectral in $x$. One of them is also spectral in $y$, while the other is of second order in $y$. We split (1.1) into two differential equations:

$$
\begin{aligned}
2 \phi_{t x}= & \left(k \phi_{x}-\frac{\gamma+1}{2} \phi_{x}^{2}\right)_{x}, \\
2 \phi_{t x}=4 \phi_{y y} &
\end{aligned}
$$

In Section 5 we discuss stability for (1.4), and in Section 6 we prove that the eigenvalues of the spatial operator for the two schemes approximating (1.5) have nonpositive real part. Stability is proved for the semidiscrete approximation of (1.5) using a spectral method in the $x$-direction and finite differencing in the $y$-direction. Similar results are proved in Section 7 for the two-dimensional heat equation.

The extension of these schemes and numerical results for high Mach numbers are given in [6] and [5]. It is shown that one may still use these schemes when shocks are present (high Mach numbers) by filtering the results. The spectral filter proposed fits the approximated solution to a sum of a step function and a truncated Chebyshev series, and thus the scheme retains spectral accuracy.

2. Derivation of the Equations and Finite Difference Methods. The small disturbance equation of transonic flow is derived by asymptotic expansion applied to the equations of gas dynamics. The small expansion parameter is the airfoil thickness ratio $\tau$ and the Mach number is assumed to be near 1 .

To first order the following equations result for the disturbed flow:

$$
2 u_{t}=\left(k u-\frac{\gamma+1}{2} u^{2}\right)_{x}+4 v_{y}, \quad v_{x}-u_{y}=0 .
$$

A velocity potential is then introduced by

$$
u=\phi_{x}, \quad v=\phi_{y},
$$

and (1.1) results. For additional details see [3], [2], [5].

We consider a bounded spatial domain $-1 \leq x, y \leq 1$, in which the airfoil is represented by

$$
y(x)=-1+\tau F(x), \quad|x|<x_{0}, \quad x_{0} \ll 1 .
$$

Assume that the boundaries $x= \pm 1, y=1$ can be viewed as far away from the airfoil, so that the disturbed flow there is zero. Then, we have

$$
\begin{aligned}
\phi(-1, y, t) & =0, \\
u(1, y, t) & =0, \\
\phi_{y}(x, 1, t) & =0 .
\end{aligned}
$$

On the airfoil the flow is tangent to the body. Since $\tau$ tends to zero in our asymptotic expansion, this condition should be applied at $y=-1,|x|<x_{0}$. Thus

$$
\phi_{y}(x,-1, t)= \begin{cases}F^{\prime}(x), & |x|<x_{0}, \\ 0, & |x|>x_{0} .\end{cases}
$$


In addition, we must supply initial conditions for (1.1):

$$
\phi(x, y, 0)=\phi_{0}(x, y)
$$

During the last decade many numerical calculations using equations (1.1) or (1.2) have been presented (see [13], [4], [1], [2], [12]). We shall describe two of them. The first is the Murman-Cole (M-C) [13] treatment of the time-independent equation, and the second is the Engquist-Osher scheme (E-O) [4] for the time-dependent and the time-independent equations.

Murman and Cole treated numerically the steady state equation. As was noted, the equation is of mixed type. At each mesh point the velocity $\phi_{x}$ is computed (approximately) to determine if the flow is supersonic $\left(\phi_{x}>k(\gamma+1)^{-1}\right)$ or subsonic $\left(\phi_{x}<k(\gamma+1)^{-1}\right)$. The appropriate hyperbolic or elliptic type of difference equation is then selected for that mesh point. The resulting large system of algebraic equations is solved iteratively by a line relaxation algorithm. Each vertical line is relaxed successively, proceeding in the positive $x$-direction. At each stage of the iteration the local velocity is tested to select an elliptic or a hyperbolic difference approximation.

It was pointed out in [4], [2] that the Murman-Cole scheme leads to nonlinear instabilities, even though the scheme should be stable according to linear stability analysis. Furthermore, it was reported in [4], [12] that the M-C scheme admits entropy-violating shocks as solutions.

Engquist and Osher [4] modified the Cole-Murman scheme so that entropyviolating shocks cannot be obtained, and presented a stability analysis for the full nonlinear problem. Define

$$
\bar{u}=\frac{k}{\gamma+1} .
$$

In regions where

$$
u_{j-1, k}, u_{j k}, u_{j+1, k}>\bar{u} \quad \text { or } \quad u_{j-1, k}, u_{j k}, u_{j+1, k}<\bar{u},
$$

the M-C differences for the point $\left(x_{j}, y_{k}\right)$ are identical to those of E-O for the timeindependent scheme. There are modifications near the surface of interference of subsonic and supersonic regions.

It was proved in [4] that this scheme, with the Courant condition

$$
\max _{u_{j k}^{n}} \lambda_{n}(x)\left|f^{\prime}\left(u_{j k}^{n}\right)\right|=\varepsilon_{0}<\frac{1}{3},
$$

is stable with respect to the norm

$$
\|u\|^{2}=\sum_{j=0}^{N} \sum_{k=0}^{M} u_{j k}^{2} \Delta x \Delta y .
$$

The scheme is of first order. If it converges to a time-independent solution, then it is second-order accurate in subsonic regions and first-order in supersonic regions. From the discussion above it appears worthwhile to look for a high-order scheme for the small disturbance problem. We focus our attention on the $x$-direction, since changes spread much more slowly in the $y$-direction. Moreover, the difficulty of developing high-order finite difference schemes is due to the type-dependent equation, for which hyperbolic or elliptic regions are determined by the coefficient 
of $\phi_{x x}$. We expect this scheme to have spectral accuracy in $x$, instead of the firstor at most second-order accuracy obtained by the finite difference schemes. Before we turn to the description of the spectral schemes (in Section 4), we next prove that the linearized small disturbance equation is well posed.

3. Well-Posedness of the Linearized Differential Equation. Consider the linearized differential equation with homogeneous boundary conditions. In Theorem 3.1 we establish the well-posedness of this problem. This is a new result, and is given to make sure that the boundary conditions (2.1)-(2.4) yield a well-posed problem.

Define $H^{p}(\Omega)$ to be the Sobolev space of functions $u$ such that $u$ and its spatial derivatives up to order $p$ are in $L_{2}(\Omega)$ and

$$
\Omega=\{x, y \mid 1 \leq x, y \leq 1\}
$$

THEOREM 3.1. Consider the problem

$$
\begin{gathered}
\phi_{t x}=-a(x, y) u_{x}+\phi_{y y}, \quad x, y \in \Omega, t \geq 0, \\
\phi(-1, y, t)=0, \\
u(1, y, t)=0, \\
\phi_{y}(x, \pm 1, t)=0, \\
\phi(x, y, 0)=\phi_{0}(x, y),
\end{gathered}
$$

where

$$
u=\phi_{x}
$$

and assume

$$
\begin{aligned}
& a( \pm 1, y) \leq 0, \\
& a(x, y) \in C^{1},
\end{aligned}
$$

and

$$
\phi_{0}(x, y) \in H^{1}(\Omega)
$$

Then (3.1)-(3.5) is a well-posed problem, i.e., there exists a constant $C$ such that

$$
\int_{-1}^{1} \int_{-1}^{1}\left[\frac{\partial \phi}{\partial x}(x, y, t)\right]^{2} d x d y \leq e^{C t} \int_{-1}^{1} \int_{-1}^{1}\left[\frac{\partial \phi_{0}}{\partial x}(x, y)\right]^{2} d x d y
$$

Proof. Multiplication of (3.1) by $\phi_{x}=u$ and integration with respect to $x, y$ over the square $-1 \leq x, y \leq 1$ yields

$$
\int_{-1}^{1} \int_{-1}^{1} \phi_{t x} \phi_{x} d x d y=-\int_{-1}^{1} \int_{-1}^{1} a(x, y) u_{x} \phi_{x} d x d y+\int_{-1}^{1} \int_{-1}^{1} \phi_{y y} \phi_{x} d x d y .
$$

Hence,

$$
\frac{1}{2} \frac{\partial}{\partial t} \int_{-1}^{1} \int_{-1}^{1} u^{2} d x d y=-\frac{1}{2} \int_{-1}^{1} \int_{-1}^{1} a(x, y) \frac{\partial}{\partial x}\left(u^{2}\right) d x d y+\int_{-1}^{1} \int_{-1}^{1} \phi_{y y} \phi_{x} d x d y
$$


We integrate by parts the first term with respect to $x$ and the second with respect to $y$. Therefore,

$$
\begin{aligned}
\frac{1}{2} \frac{\partial}{\partial t} \int_{-1}^{1} \int_{-1}^{1} u^{2} d x d y= & \int_{-1}^{1}\left[-\frac{1}{2} u^{2} a(x, y)\right]_{x=-1}^{x=1} d y+\frac{1}{2} \int_{-1}^{1} \int_{-1}^{1} u^{2} \frac{\partial a}{\partial x} d x d y \\
& +\int_{-1}^{1}\left[\phi_{x} \phi_{y}\right]_{y=-1}^{y=1} d x-\int_{-1}^{1} \int_{-1}^{1} \phi_{x y} \phi_{y} d x d y
\end{aligned}
$$

Invoking (3.3), (3.7) and (3.5), we find

$$
\frac{1}{2} \frac{\partial}{\partial t} \int_{-1}^{1} \int_{-1}^{1} u^{2} d x d y=+\frac{1}{2} \int_{-1}^{1} \int_{-1}^{1} u^{2} \frac{\partial a}{\partial x} d x d y-\frac{1}{2} \int_{-1}^{1} \int_{-1}^{1} \frac{\partial}{\partial x}\left(\phi_{y}^{2}\right) d x
$$

By (3.8),

$$
\left|\frac{\partial a}{\partial x}\right| \leq C
$$

Hence, (3.10) yields

$$
\frac{1}{2} \frac{\partial}{\partial t} \int_{-1}^{1} \int_{-1}^{1} u^{2} d x d y \leq \frac{1}{2} C \int_{-1}^{1} \int_{-1}^{1} u^{2} d x d y-\frac{1}{2} \int_{-1}^{1}\left(\phi_{y}^{2}\right)_{x=-1}^{x=1} d y .
$$

By $(3.2),\left.\phi_{y}\right|_{x=-1}=0$, and therefore

$$
\begin{aligned}
\frac{1}{2} \frac{\partial}{\partial t} \int_{-1}^{1} \int_{-1}^{1} u^{2} d x d y & \leq \frac{1}{2} C \int_{-1}^{1} \int_{-1}^{1} u^{2} d x d y-\frac{1}{2} \int_{-1}^{1} \phi_{y}^{2}(1, y, t) d y \\
& \leq \frac{1}{2} C \int_{-1}^{1} \int_{-1}^{1} u^{2} d x d y
\end{aligned}
$$

or

$$
\int_{-1}^{1} \int_{-1}^{1} u^{2}(x, y, t) d x d y \leq e^{C t} \int_{-1}^{1} \int_{-1}^{1}\left[\frac{\partial}{\partial x} \phi_{0}(x, y)\right]^{2} d x d y
$$

4. The Numerical Scheme. We now describe our spectral scheme for (1.1), (2.1)-(2.5). As in [4], we split the problem (1.1), (2.1)-(2.5) into two differential problems. The first one is

$$
\begin{gathered}
u_{t}=-(f(u))_{x} \\
u(1, y, t)=0
\end{gathered}
$$

where $u=\phi_{x}$. Observe that (4.1) is in conservative form. Omitting the factor two, which appears next to $\phi_{y y}$, the second problem is

$$
\begin{gathered}
\phi_{t x}=\phi_{y y}, \\
\phi(-1, y, t)=0, \\
\phi_{y}(x, \pm 1, t)=F_{ \pm}(x),
\end{gathered}
$$

where $F_{+}(x)=0$, and

$$
F_{-}(x)= \begin{cases}F^{\prime}(x), & |x|<x_{0} \\ 0, & |x|>x_{0}\end{cases}
$$

(see (2.3) and (2.4)). Note that $F(x)$ describes the shape of the airfoil. Both of the above problems must be supplied with initial conditions. 
(a) Discretization in Time. Since we are interested in the steady state only, we discretize $u_{t}$ in (4.1) or (4.3) using finite differences. One may present both problems above in the form

$$
u_{t}=G(u)
$$

For the first one,

and for the second,

$$
G(u)=G_{1}(u)=-\frac{\partial}{\partial x} f(u)
$$

$$
G(u)=G_{2}(u)=\int_{-1}^{x} \frac{\partial^{2} u}{\partial y^{2}} d x .
$$

We apply the modified Euler scheme

$$
u^{n+1 / 2}=u^{n}+\frac{\Delta t}{2} G\left(u^{n}\right), \quad u^{n+1}=u^{n}+\Delta t G\left(u^{n+1 / 2}\right) .
$$

Let $L(\Delta t)$ be the operator which acts on $u^{n}$ to yield $u^{n+1}$ :

$$
L(\Delta t) u^{n}=u^{n+1}=u^{n}+\Delta t G\left(u^{n}+\frac{\Delta t}{2} G\left(u^{n}\right)\right) .
$$

$L_{1}(\Delta t), L_{2}(\Delta t)$ are defined as $L(\Delta t)$ with $G_{1}, G_{2}$ replacing $G$. According to [8], the following discretization in time for (1.1),

$$
u^{n+1}=L_{1}\left(\frac{\Delta t}{2}\right) L_{2}\left(\frac{\Delta t}{2}\right) L_{2}\left(\frac{\Delta t}{2}\right) L_{1}\left(\frac{\Delta t}{2}\right) u^{n},
$$

is second-order accurate in the time variable, even in the nonlinear case. Since this scheme is second-order accurate in time, one can use it to approximate the time-dependent solution as well, and if higher-order accuracy in time is desired, one may use higher-order Runge-Kutta schemes. Spectrally accurate stepping in time was suggested by Tal-Ezer [16] for linear problems. This cannot be applied here because of the nonlinearity of the problem (1.1).

(b) Discretization in Space. In both problems (4.1)-(4.2) and (4.3)-(4.5), derivatives or integrals with respect to the spatial variables $x$ or $y$ appear. It is sufficient to describe how we discretize $\partial u / \partial x$ and $\int_{-1}^{x} u(\tau) d \tau$.

Let $P_{N} u$ be the Chebyshev pseudospectral projection of $u$ on the subspace of polynomials of degree $N$ or less, i.e.,

$$
u_{N}(x, y)=P_{N} u(x, y)=\sum_{n=0}^{N} a_{n}(y) T_{n}(x)
$$

where

$$
u_{N}\left(x_{i}, y\right)=u\left(x_{i}, y\right), \quad x_{i}=\cos \frac{\pi i}{N}, \quad 0 \leq i \leq N
$$

We discretize $\partial / \partial x$ by differentiating $P_{N} u$, and then denote the resulting operator by $L_{N}$. Hence

$$
L_{N} u=P_{N} \frac{\partial}{\partial x} P_{N} u=\sum_{n=0}^{N} a_{n}(y) T_{n}^{\prime}(x)=\sum_{n=0}^{N} b_{n}(y) T_{n}(x)
$$

where

$$
b_{N}(y)=0, \quad b_{N-1}(y)=2 N a_{N}(y)
$$


and

$$
\begin{gathered}
\bar{c}_{k} b_{k}(y)=b_{k+2}(y)+2(k+1) a_{k+1}(y), \quad 0 \leq k \leq N-2, \\
\bar{c}_{0}=\bar{c}_{N}=2,
\end{gathered}
$$

and

$$
\bar{c}_{j}=1, \quad 1 \leq j \leq N-1 .
$$

We apply $L_{N} u$ for $y=y_{j}=\cos \pi j / M, 0 \leq j \leq M$.

Next, integration is done in a similar way,

$$
\begin{aligned}
I_{N} & =P_{N} \int_{-1}^{x} P_{N} u d \tau=P_{N} \sum_{n=0}^{N} a_{n}(y) \int_{-1}^{x} T_{N}(\tau) d \tau \\
& =P_{N} \sum_{n=0}^{N+1} d_{n}(y) T_{n}(x) .
\end{aligned}
$$

By integrating the recurrence formula

$$
2 T_{n}(x)=\frac{T_{n+1}^{\prime}(x)}{n+1}+\frac{T_{n-1}^{\prime}(x)}{n-1}
$$

we find

$$
\begin{array}{ccrl}
d_{N+1}=\frac{a_{N}}{2(N+2)}, & d_{N}=\frac{a_{N-1}}{2(N+1)}, \\
d_{n}=\frac{1}{2} \frac{a_{n-1}-a_{n+1}}{n}, & 3 \leq n \leq N-1, \\
d_{2}=\frac{a_{1}}{4}-\frac{a_{3}}{4}, & d_{1}=a_{0}-\frac{a_{2}}{2},
\end{array}
$$

and we choose $d_{0}$ such that

$$
\sum_{n=0}^{N+1} d_{n}(y) T_{n}(-1)=0 .
$$

We shall consider two types of schemes for the discretization of $\partial^{2} / \partial y^{2}$. The first is spectral in $y$ (scheme A), and the second is a finite difference one (scheme B). It is reasonable to use the latter, since for the transonic problems changes spread much more slowly in the $y$-direction than in the $x$-direction.

(b1) Spectral Approximation in y (scheme A). As described above, we discretize $\partial^{2} / \partial y^{2}$ by a Chebyshev pseudospectral method. For a fixed $x$, define $P_{M} u(x, y)$ to be the Chebyshev pseudospectral projection of $u(x, y)$ onto the subspace of polynomials of degree less than or equal to $M$, i.e.,

$$
P_{M} u=\sum_{m=0}^{M} e_{m}(x) T_{m}(y)
$$

Define $D_{M}^{(1)}(y)$ as follows:

$$
\begin{aligned}
D_{M}^{(1)}(y) & =P_{M} \frac{\partial^{2}}{\partial y^{2}} P_{M} u(x, y)=\sum_{m=0}^{M} e_{m}(x) T_{m}^{\prime \prime}(y) \\
& =\sum_{m=0}^{M} f_{m}(x) T_{m}(y),
\end{aligned}
$$


where (see $[10$, p. 16])

$$
f_{M}=f_{M-1}=0, \quad c_{m} f_{m}=\sum_{\substack{p=m+2 \\ p+m \text { even }}}^{M} p\left(p^{2}-m^{2}\right) a_{p}, \quad 0 \leq m \leq M-2,
$$

and

$$
c_{0}=2, \quad c_{m}=1, \quad 1 \leq m \leq M-2 .
$$

We are now ready to present our semidiscrete spectral scheme for (4.1)-(4.5). Denote by $U$ the approximation to $u$ and by $\Phi$ the approximation to $\phi$, where

$$
\Phi=I_{N} U \text {. }
$$

The semidiscrete approximation to (4.1)-(4.2) is

$$
\begin{gathered}
\frac{\partial U}{\partial t}=-L_{N}(x) f(U), \\
U(-1, y, t)=0,
\end{gathered}
$$

and for (4.3)-(4.5) we have the following scheme:

Scheme A.

$$
\begin{gathered}
\frac{\partial \Phi}{\partial t}=I_{N} D_{M}^{(1)}(y) \Phi \\
L_{M}(y) \Phi(x, \pm 1, t)=P_{N} F_{ \pm}(x) .
\end{gathered}
$$

This scheme has spectral accuracy in the space variables.

(b2) Finite Difference Approximation in y (scheme B). Using finite differences for approximating $\partial^{2} / \partial y^{2}$, we have

$$
\frac{\partial^{2}}{\partial y^{2}} u \approx D_{M}^{(2)}(y) u(x, y)=\frac{u\left(x, y_{j+1}\right)-2 u\left(x, y_{j}\right)+u\left(x, y_{j-1}\right)}{(\Delta y)^{2}}
$$

where $\Delta y=2 / M, y_{j}=1-(\Delta y) \cdot j, 1 \leq j \leq M-1$. We apply $D_{M}^{(2)}(y)$ for $x=x_{i}=\cos \pi i / N, 0 \leq i \leq N-1$.

To conclude, for (4.1)-(4.2) we use the same discretization as in (4.9)-(4.10) and for (4.3)-(4.5) and we obtain the following semidiscrete scheme:

Scheme B.

$$
\begin{gathered}
\frac{\partial \Phi}{\partial t}=I_{N}(x) D_{M}^{(2)}(y) \Phi, \\
\frac{\Phi\left(x_{i}, 1\right)-\Phi\left(x_{i}, 1-\Delta y\right)}{\Delta y}=F_{+}\left(x_{i}\right), \quad 0 \leq i \leq N-1, \\
\frac{\Phi\left(x_{i},-1+\Delta y\right)-\Phi\left(x_{i},-1\right)}{\Delta y}=F_{-}\left(x_{i}\right), \quad 0 \leq i \leq N-1,
\end{gathered}
$$

where $\Phi=I_{N} U$. This scheme has spectral accuracy in $x$ and is of second order in $y$.

The reason we have looked for other schemes rather than the spectral one for approximating $\partial^{2} / \partial y^{2}$ is the asymptotic behavior of the eigenvalues of $D_{M}^{(1)}(y)$, where

$$
D_{M}^{(1)}(y)=P_{M} \frac{\partial^{2}}{\partial y^{2}} P_{M}
$$


The largest eigenvalue of $D_{M}^{(1)}(y)$ grows like $O\left(M^{4}\right)$ when $M$ grows to infinity (see $[10$, p. 115]). This implies a restriction on $\Delta t$ of the form

$$
\Delta t=O\left(\frac{1}{M^{4}}\right) .
$$

For small $M$ ( 8 or 16$)$ we may still use spectral differentiation with respect to $y$ and get spectral accuracy. But if we wish to increase $M$, it is preferable to use a finite difference approximation, in which

$$
\Delta t=O\left(\frac{1}{M^{2}}\right)
$$

An alternative is to use an implicit scheme for the time discretization (see $[10$, Section 9]). In this case the scheme is unconditionally stable but one should solve a full system of linear equations every time step. Research is being done on this subject; see for example [14].

5. The $y$-Independent Problem. In this section we consider stability questions for the semidiscrete approximation (4.9)-(4.10) of the problem (4.1)-(4.2). We rewrite (4.1) in the form

$$
u_{t}+a(u) u_{x}=0
$$

where $a(u)=\partial f / \partial u$. In [7] there is a stability proof for the linear case $a(u)=-1$, with the boundary condition $u(1, y, t)=0$. A similar stability result is valid for the case $a(u)=1$ with $u(-1, y, t)=0$, or even in a more general case, as long as $a(u)=a(x)$ does not change sign. In such a proof, one obtains energy estimates for $u(x) / \sqrt{|a(x)|}$ rather than for $u(x)$. However, there is no proof for the general linear case. Stability of the Chebyshev pseudospectral approximation for two specific variable coefficients problems was proved by D. Gottlieb [7]. In numerical calculations for more general problems there were no reports of instabilities, as long as there was no shock involved.

Consider the full discrete approximation to (4.1)-(4.2),

$$
\begin{aligned}
& U^{n+1 / 2}=U^{n}+\frac{\Delta t}{2} L_{N} f\left(U^{n}\right), \\
& U^{n}=U^{n}+\Delta t L_{N} f\left(U^{n+1 / 2}\right) .
\end{aligned}
$$

Since the spectral radius of $L_{N}$ is $O\left(N^{2}\right)$ (see [10, p. 90]),

$$
\Delta t=O\left(\frac{1}{N^{2}}\right) \text {. }
$$

By a numerical calculation of the spectral radius of $L_{N}$ it was found ([10, p. 110]) that the scheme is stable if

$$
\Delta t \max |a(u)| \leq \frac{8}{N^{2}} .
$$

Note that the Chebyshev collocation points $x_{i}=\cos \pi i / N, i=0, \ldots, N$, are more crowded near the boundaries $x= \pm 1$. This allows us to improve our polynomial approximation, since it counters the tendency of polynomials to oscillate with large amplitude near the boundaries ([15]). For our problem an airfoil is located at $|x| \leq x_{0}$, and the tips of the airfoil $x= \pm x_{0}$ may cause large gradients of the 
solution to appear near these points (see (2.4)). We therefore divide the region $-1 \leq x \leq 1$ into three parts: $-1 \leq x \leq-x_{0},-x_{0} \leq x \leq x_{0}, x_{0} \leq x \leq 1$, and thus have more collocation points near the tips of the body. We prove that the scheme remains stable under these conditions.

Consider the model problem

$$
\begin{gathered}
\omega_{t}=\omega_{x}, \quad-1 \leq x \leq 3, \\
\omega(3, t)=g(t) .
\end{gathered}
$$

We divide the domain into two subdomains $-1 \leq x \leq 1,1 \leq x \leq 3$ and apply pseudospectral methods to each domain. The two polynomials $u_{N}^{\mathrm{I}}, u_{N}^{\mathrm{II}}$ of degree $N$ satisfy

$$
\begin{array}{cc}
\frac{\partial u_{N}^{\mathrm{I}}}{\partial t}=\frac{\partial u_{N}^{\mathrm{I}}}{\partial x}, & x=x_{j}^{(1)}=2+\cos \frac{\pi j}{N}, \quad j=1, \ldots, N-1, \\
\frac{\partial u_{N}^{\mathrm{II}}}{\partial t}=\frac{\partial u_{N}^{\mathrm{II}}}{\partial x}, & x=x_{j}^{(2)}=\cos \frac{\pi j}{N}, \quad j=1, \ldots, N-1, \\
u_{N}^{\mathrm{I}}(1, t)=g(t), &
\end{array}
$$

and the continuity equation

$$
u_{N}^{\mathrm{I}}(1, t)=u_{N}^{\mathrm{II}}(1, t)
$$

Consider the problem

$$
\begin{gathered}
u_{t}=u_{x}, \quad-1 \leq x \leq 1, \\
u(1, t)=g(t),
\end{gathered}
$$

and denote its pseudospectral approximation by $u_{N}$. Define

$$
V_{N}=u_{N}-g(t) \frac{T_{N}^{\prime}(x)}{N^{2}}
$$

which yields $V_{N}(1, t)=0$.

Gottlieb and Turkel [11] derived an energy inequality for $V_{N}$ :

$$
\frac{1}{2}\left\|V_{N}(t)\right\|^{2}+\frac{\omega_{N}}{4} \int_{0}^{0} V_{N}^{2}\left(x_{N}, \tau\right) d \tau \leq K N \int_{0}^{t} g^{2}(\tau) d \tau+\frac{1}{2}\left\|V_{N}(0)\right\|^{2},
$$

where

$$
\begin{gathered}
\|v\|^{2}=\sum_{j=0}^{N-1} v^{2}\left(x_{j}\right) \hat{\omega}_{j}, \quad x_{j}=\cos \frac{\pi j}{N}, \\
\hat{\omega}_{j}=\left(1+x_{j}\right) \omega_{j}, \\
\omega_{j}=\frac{\pi}{N}, \quad 1 \leq j \leq N-1, \quad \omega_{0}=\omega_{n}=\frac{1}{2} \frac{\pi}{N} .
\end{gathered}
$$

We now apply (5.3) to $u_{N}^{\mathrm{I}}$ and $u_{N}^{\mathrm{II}}$. Define

$$
\begin{aligned}
& V_{N}^{\mathrm{I}}=u_{N}^{\mathrm{I}}-g(t) \cdot \frac{T_{N}^{\prime}(x-2)}{N^{2}} \\
& V_{N}^{\mathrm{II}}=u_{N}^{\mathrm{II}}-u_{N}^{\mathrm{I}}(1, t) \frac{T_{N}^{\prime}(x)}{N^{2}}
\end{aligned}
$$


By (5.3),

$$
\begin{aligned}
& \frac{1}{2}\left\|V_{N}^{\mathrm{I}}(t)\right\|_{1}^{2}+\frac{\omega_{N}}{4} \int_{0}^{t}\left[V_{N}^{\mathrm{I}}(1, \tau)\right]^{2} d \tau \\
& \leq K N \int_{0}^{t} g^{2}(\tau) d \tau+\frac{1}{2}\left\|V_{N}^{\mathrm{I}}(0)\right\|_{1}^{2}
\end{aligned}
$$

$$
\begin{aligned}
& \frac{1}{2}\left\|V_{N}^{\mathrm{II}}(t)\right\|_{2}^{2}+\frac{\omega_{N}}{4} \int_{0}^{t}\left[V_{N}^{\mathrm{II}}(-1, \tau)\right]^{2} d \tau \\
& \leq K N \int_{0}^{t}\left[u_{N}^{\mathrm{I}}(1, \tau)\right]^{2} d \tau+\frac{1}{2}\left\|V_{N}^{\mathrm{II}}(0)\right\|_{2}^{2}
\end{aligned}
$$

In (5.7) and (5.8),

$$
\|v\|_{1,2}^{2}=\sum_{j=0}^{N-1} v^{2}\left(x_{j}^{(1),(2)}\right) \hat{\omega}_{j}^{(1),(2)}
$$

where $x_{j}^{(1),(2)}$ are defined in (5.1) and

$$
\hat{\omega}_{j}^{(1)}=\omega_{j} \cdot\left(x_{j}^{(1)}-1\right), \quad \hat{\omega}_{j}^{(2)}=\omega_{j} \cdot\left(x_{j}^{(2)}+1\right),
$$

with $\omega_{j}$ defined in (5.4).

Summing (5.7) and (5.8), we obtain

$$
\begin{aligned}
& \frac{1}{2}\left\|V_{N}(t)\right\|_{1+2}^{2}+\frac{\omega_{N}}{4} \int_{0}^{t}\left[V_{N}^{\mathrm{I}}(1, \tau)\right]^{2} d \tau+\frac{\omega_{N}}{4} \int_{0}^{t}\left[V_{N}^{\mathrm{II}}(-1, \tau)\right]^{2} d \tau \\
& \leq K N \int_{0}^{t} g^{2}(\tau) d \tau+K N \int_{0}^{t}\left[u_{N}^{\mathrm{I}}(1, \tau)\right]^{2} d \tau+\frac{1}{2}\left\|V_{N}(0)\right\|_{1+2}^{2}
\end{aligned}
$$

where

$$
\left\|V_{N}(t)\right\|_{1+2}^{2}=\left\|V_{N}^{\mathrm{I}}\right\|_{1}^{2}+\left\|V_{N}^{\mathrm{II}}\right\|_{2}^{2}
$$

Invoking (5.5), we find

$$
u_{N}^{\mathrm{I}}(1, \tau)=V_{N}^{\mathrm{I}}(1, \tau)+g(\tau) \frac{T_{N}^{\prime}(-1)}{N^{2}} .
$$

Hence,

$$
\left[u_{N}^{\mathrm{I}}(1, \tau)\right]^{2} \leq 2\left\{\left[V_{N}^{\mathrm{I}}(1, \tau)\right]^{2}+g^{2}(\tau)\right\} .
$$

Using (5.7) and (5.10), we conclude that

$$
\begin{aligned}
\frac{\omega_{N}}{4} \int_{0}^{t}\left[u_{N}^{\mathrm{I}}(1, \tau)\right]^{2} d \tau \leq \frac{\omega_{N}}{2} \int_{0}^{t}\left[V_{N}^{\mathrm{I}}(1, \tau)\right]^{2} d \tau+\frac{\omega_{N}}{2} \int_{0}^{t} g^{2}(\tau) d \tau \\
\quad \leq\left(2 K N+\frac{\omega_{N}}{2}\right) \int_{0}^{t} g^{2}(\tau) d \tau+\left\|V_{N}^{\mathrm{I}}(0)\right\|_{1}^{2} \\
\quad \leq\left(2 K N+\frac{\omega_{N}}{2}\right) \int_{0}^{t} g^{2}(\tau) d \tau+\left\|V_{N}(0)\right\|_{1+2}^{2} .
\end{aligned}
$$


Substitution of the above inequality into (5.9) yields

$$
\begin{aligned}
\frac{1}{2}\left\|V_{N}(t)\right\|_{1+2}^{2}+\frac{\omega_{N}}{4} \int_{0}^{t}\left[V_{N}^{\mathrm{II}}(-1, \tau)\right]^{2} d \tau \\
\leq K N \int_{0}^{t} g^{2}(\tau) d \tau \\
\quad+\frac{4 K N}{\omega_{N}}\left[2 K N \int_{0}^{1} g^{2}(\tau) d \tau+\left\|V_{N}(0)\right\|_{1+2}^{2}+\frac{\omega_{N}}{2} \int_{0}^{1} g^{2}(\tau) d \tau\right] \\
\quad+\frac{1}{2}\left\|V_{N}(0)\right\|_{1+2}^{2},
\end{aligned}
$$

or

$$
\begin{aligned}
& \frac{1}{2}\left\|V_{N}(t)\right\|_{1+2}^{2}+\frac{\omega_{N}}{4} \int_{0}^{t}\left[V_{N}^{\mathrm{II}}(-1, \tau)\right]^{2} d \tau \\
& \quad \leq K_{1} N^{3} \int_{0}^{1} g^{2}(\tau) d \tau+\frac{1}{2} K_{2} N^{2}\left\|V_{N}(0)\right\|_{1+2}^{2} .
\end{aligned}
$$

Thus, we proved that this procedure is algebraically stable (see [10, Section 5]), i.e., the constant appearing next to $\left\|V_{N}(0)\right\|_{1+2}^{2}$ is bounded by $N^{2 r}$ for some finite $r$. It was shown in [10] that if a scheme is algebraically stable and if its truncation error is spectrally small (i.e., less than $N^{-p}$ for $p>r$ ), then the scheme is convergent.

6. The $y$-Dependent Problem. In this section we consider the problem (4.3)(4.5). Attention is restricted to the case $F_{ \pm}(x)=0$, i.e., homogeneous boundary conditions:

$$
\begin{gathered}
\phi_{t x}=\phi_{y y}, \\
\phi(-1, y, t)=0, \\
\phi_{y}(x, \pm 1, t)=0, \\
\phi(x, y, 0)=\phi_{0}(x, y) .
\end{gathered}
$$

The solution of the problem involving inhomogeneous boundary conditions is a sum of an arbitrary function having the imposed boundary values and a solution to a perturbed problem $\left(6.1^{\prime}\right),(6.2)-(6.4)$, where

$$
\phi_{t x}=\phi_{y y}+g(x, y) \text {. }
$$

One can represent the solution of $\left(6.1^{\prime}\right)$ in the form

$$
\phi=e^{G t} \phi_{0}+\int_{0}^{t} e^{G(t-s)} g(x, y) d s, \quad \text { where } G(u)=\int_{-1}^{x} \frac{\partial^{2} u}{\partial y^{2}} d x .
$$

This representation was used in the proof of the equivalence theorem in $[10, p$. 47 ], in which it was proved that consistent stable schemes for $\left(6.1^{\prime}\right)$ are convergent. Note that a scheme is stable $([10$, p. 47$])$ if $\left\|e^{G_{N M} t}\right\| \leq K(t)$, where $G_{N M}$ is an approximation to $G$, and therefore stability is a feature of the homogeneous equation (6.1) and is not affected by the nonhomogeneous term $g(x, y)$. We therefore treat the homogeneous equation (6.1) with the homogeneous boundary conditions (6.2)(6.4). The problem (6.1)-(6.4) is well posed, as one may check in a way similar to the proof of Theorem 3.1, omitting the term $a(x, y) u_{x}$. 
Next, consider semidiscrete approximations to (6.1)-(6.4), i.e., time is a continuous variable. Two types of approximation are considered.

(a) Pseudospectral in spatial variables, i.e., the scheme is described by (4.11)(4.12) (scheme A).

(b) Pseudospectral in $x$ and finite differences in $y$. The scheme is presented in (4.13)-(4.15) with $F_{ \pm}(x)=0$ (scheme B).

Recall again the following representation of (6.1),

$$
\phi_{t}=\int_{-1}^{x} \phi_{y y} d x
$$

or equivalently,

$$
\phi_{t}=G \phi, \quad G \phi=\int_{-1}^{x} \phi_{y y} d x .
$$

In the previous section we described two ways of approximating $G$. Denote by $G_{N M}$ the approximate operator. We shall inquire whether the approximate solution $\Phi$ does not grow as $t \rightarrow \infty$. In other words we shall prove that the eigenvalues of $G_{N M}$ have a nonpositive real part.

(a) The Spectrum of the Spatial Operator of Scheme A. To investigate the eigenvalues of $G_{N M}$, we seek solutions of the form $e^{\lambda t} \Phi_{N M}(x, y)$ for (4.11), where $\Phi_{N M}$ is a polynomial of degree $N$ in $x$ and $M$ in the $y$ variable. Thus, up to a spectral error we may treat the equation

$$
\begin{aligned}
\lambda L_{N} \Phi_{N M}(x, y) & =D_{M}^{(1)}(y) \Phi_{N M}(x, y), \\
\text { at } x & =x_{i}=\cos \pi \frac{i}{N}, \quad 0 \leq i \leq N-1, \\
y & =y_{j}=\cos \pi \frac{j}{M}, \quad 1 \leq j \leq M-1 .
\end{aligned}
$$

In order to prove that $\operatorname{Re}(\lambda) \leq 0$, we represent $\Phi_{N M}(x, y)$ as a sum of $M-1$ functions, denoted by $\left\{\psi_{M}^{k}(y)\right\}_{k=1}^{M-1}$, which are the eigenfunctions of $D_{M}^{(1)}(y)$ satisfying $\left(\partial \psi_{M}^{k} / \partial y\right)(y= \pm 1)=0$. In the following lemma we prove that there are such $M-1$ independent functions, and therefore the desired representation is possible.

LEMMA 6.1. Let $\psi_{M}(y, \lambda)$ be an eigenfunction of the problem

$$
\begin{gathered}
\lambda \psi_{M}(y, \lambda)=D_{M}^{(1)}(y) \psi_{M}(y, \lambda)+(A+B y) T_{M}^{\prime}(y), \\
\frac{\partial \psi_{M}}{\partial y}( \pm 1, \lambda)=0,
\end{gathered}
$$

where $\psi_{M}(y, \lambda)$ is a polynomial of degree less than or equal to $M$. Then there are $M-1$ independent functions satisfying (6.5)-(6.6).

Proof. We prove that if

$$
\sum_{i=1}^{M-1} \alpha_{i} \psi_{M}\left(y, \lambda_{i}\right)=0
$$

then $\alpha_{i}=0, i=1, \ldots, M-1$. By $(6.7)$,

$$
\sum_{i=1}^{M-1} \alpha_{i} \psi_{M}\left(y_{k}, \lambda_{i}\right)=0, \quad y_{k}=\cos (\pi k / M), \quad k=1, \ldots, M-1
$$


The vectors

$$
\left(\begin{array}{c}
\psi_{M}\left(y_{1}, \lambda_{i}\right) \\
\vdots \\
\psi_{M}\left(y_{M-1}, \lambda_{i}\right)
\end{array}\right)_{i=1, \ldots, M-1}
$$

are linearly independent since they correspond to distinct eigenvalues (by [9]), and therefore $\alpha_{i}=0$ for $i=1, \ldots, M-1$.

Remark. We shall later make use of the independence of the vectors

$$
\left(\begin{array}{c}
\psi_{M}\left(y_{1}, \lambda_{i}\right) \\
\vdots \\
\psi_{M}\left(y_{M-1}, \lambda_{i}\right)
\end{array}\right)_{i=1, \ldots, M-1}
$$

which follows from the proof of Lemma 6.1.

We are now ready to state one of the three main results of this paper, which are Theorems 6.2-6.4.

THEOREM 6.2. Let $\lambda$ be an eigenvalue of the problem

$$
\begin{gathered}
\lambda L_{N}(x) \Phi_{N M}(x, y, \lambda)=D_{M}^{(1)}(y) \Phi_{N M}(x, y, \lambda), \\
\text { for } x=x_{i}=\cos \frac{\pi i}{N}, \quad i=0, \ldots, N-1, \\
y=y_{j}=\cos \frac{\pi j}{M}, \quad j=1, \ldots, M-1, \\
\Phi_{N M}(-1, y, \lambda)=0, \\
\frac{\partial}{\partial y} \Phi_{N M}(x, \pm 1, y)=0
\end{gathered}
$$

where $L_{N}(x), D_{M}^{(1)}(y)$ are defined in (4.7) and (4.8), respectively.

Then

$$
\operatorname{Re}(\lambda) \leq 0
$$

Proof. $\Phi_{N M}(x, y, \lambda)$ satisfies $(6.10)$, so by Lemma 6.1 it may be represented in the form

$$
\Phi_{N M}(x, y, \lambda)=\sum_{k=1}^{M-1} a_{k}(x, \lambda) \Psi_{k}(y),
$$

where $a_{k}(x, \lambda)$ are polynomials of degree $N$ or less. Invoking (6.11) and (6.8), we find

$$
\begin{aligned}
\lambda \sum_{k=1}^{M-1} L_{N} a_{k}(x, \lambda) \Psi_{k}(y) & =\sum_{k=1}^{M-1} a_{k}(x, \lambda) \Psi_{k}^{\prime \prime}(y) \\
& =\sum_{k=1}^{M-1} \mu_{k} a_{k}(x, \lambda) \Psi_{k}(y), \\
\text { for } x & =x_{i}=\cos \frac{\pi i}{N}, \quad i=0, \ldots, N-1, \\
y & =y_{j}=\cos \frac{\pi j}{M}, \quad j=1, \ldots, M-1 .
\end{aligned}
$$


$\mu_{k}$ are the eigenvalues of $(6.5)-(6.6)$. Hence,

$$
\begin{aligned}
& \sum_{k=1}^{M-1}\left(\lambda L_{N} a_{k}(x, \lambda)-\mu_{k} a_{k}(x, \lambda)\right) \Psi_{k}(y)=\sum_{k=1}^{M-1} d_{k}(x) \Psi_{k}(y)=0 \\
& \text { for } x=x_{i}, y=y_{j} .
\end{aligned}
$$

Since the vectors

$$
\left(\begin{array}{c}
\Psi_{k}\left(y_{1}\right) \\
\vdots \\
\Psi_{k}\left(y_{M-1}\right)
\end{array}\right)_{k=1, \ldots, M-1}
$$

are linearly independent (see the remark after Lemma 6.1), we have

$$
d_{k}(x)=0 \quad \text { for } x=x_{i}, \quad i=0, \ldots, N-1, k=1, \ldots, M-1 .
$$

Hence,

$$
\lambda L_{N} a_{k}(x, \lambda)-\mu_{k} a_{k}(x, \lambda)=0, \quad x=x_{i}, i=0, \ldots, N-1,
$$

and by (6.9)

$$
a_{k}(-1, \lambda)=0 .
$$

We shall prove that the eigenvalues of $(6.12)-(6.13)$ have nonpositive real part. Consider the problem

$$
\begin{gathered}
\frac{\partial u_{N}}{\partial t}+\frac{\partial u_{N}}{\partial x}=0, \quad x=x_{i}, i=0, \ldots, N-1, \\
u_{N}(-1, t)=0 .
\end{gathered}
$$

In [7] stability was proved for the above problem and therefore the eigenvalues $\nu$ of the following problem

$$
\begin{gathered}
\nu U_{N}(x, \nu)+L_{N} U_{N}(x, \nu)=0, \quad x=x_{i}, i=0, \ldots, N-1, \\
U_{N}(-1, \nu)=0,
\end{gathered}
$$

have nonpositive real part. Returning to (6.12)-(6.13), we exclude the case $\lambda=0$, since this yields $\mu_{k}=0$, and we have seen that the eigenvalues of $(6.5)-(6.6)$ are negative. Hence

$$
\operatorname{Re}\left(-\frac{\mu_{k}}{\lambda}\right) \leq 0
$$

Since $\mu_{k}$ is real and negative, $\operatorname{Re}(\lambda) \leq 0$.

Numerical Evidence for Theorem 6.2. The eigenvalues $\mu_{k}$ of (6.5)-(6.6) for $M=8$ were calculated and were found to be:

$$
-2.467,-9.870,-22.185,-40.526,-54.945,-201.604,-214.372 \text {. }
$$

As proved in [9], they are real, distinct and negative. We now display $1 / \nu_{l}$, where $\nu_{l}$ are the eigenvalues of $(6.14)-(6.15)$ for $N=8$ :

$$
(-0.0312, \pm 0.114),(-0.083, \pm 0.148),(-0.165, \pm 0.124),(-0.220, \pm 0.048) .
$$

They have nonpositive real parts. The eigenvalues of $(6.8)-(6.10)$ were found numerically to be multiples of the above, i.e., $\lambda_{k l}=-\mu_{k} / \nu_{l}$, and therefore have 
nonpositive real parts. Similar results were found for the eigenvalues of (6.16)(6.18).

(b) The Spectrum and the Stability of the Spatial Operator of Scheme B. Approximating (6.1)-(6.4) by (4.13)-(4.15), we seek again solutions of the form

$$
e^{\lambda t} \Phi_{N M}(x, y)
$$

We shall prove that $\operatorname{Re}(\lambda) \leq 0$. Since Fourier analysis is used in $y$, we consider the region $-\infty \leq y \leq \infty$.

THEOREM 6.3. Let $\lambda$ be an eigenvalue of the problem

$$
\begin{gathered}
\lambda L_{N}(x) \Phi_{N M}(x, y, \lambda)=D_{M}^{(2)}(y) \Phi_{N M}(x, y, \lambda), \\
\text { for } x=x_{i}=\cos \frac{\pi j}{N}, \quad i=0, \ldots, N-1, \\
y=y_{j}=j \Delta y, \quad-\infty \leq j \leq \infty, \\
\Delta y=\frac{2}{M}, \\
\Phi_{N M}(-1, y, \lambda)=0 \\
\frac{\partial \Phi_{N M}}{\partial y}(x, \pm 1, \lambda)=0 .
\end{gathered}
$$

Then

$$
\operatorname{Re}(\lambda) \leq 0
$$

Proof. By taking the Fourier Transform of (6.16) with respect to $y$ we find

$$
\begin{aligned}
\lambda L_{N} \phi(x, \xi, \lambda) & =-4 \frac{\sin ^{2} \xi \Delta y}{(\Delta y)^{2}} \phi(x, \xi, \lambda), \\
\text { at } x & =x_{i}, \quad i=0, \ldots, N-1,
\end{aligned}
$$

$$
\phi(-1, \xi, \lambda)=0,
$$

where $\phi(x, \xi, \lambda)$ is the Fourier transform of $\Phi_{N M}(x, y, \lambda)$. We may carry on the proof as in Theorem 6.2. The only difference is that for (6.19) we have

$$
-4 \frac{\sin ^{2} \xi \Delta y}{(\Delta y)^{2}}
$$

instead of $\mu_{k}$ appearing in (6.12), and in the latter we used only the negative sign of $\mu_{k}$. When $\xi=0$, then $\lambda=0$, and therefore $\operatorname{Re}(\lambda) \leq 0$.

For scheme B we can prove stability as well.

THEOREM 6.4. Consider the problem

$$
\begin{gathered}
\frac{\partial L_{N} \Phi}{\partial t}=D_{M}^{(2)}(y) \Phi, \\
x=x_{i}=\cos \frac{\pi j}{N}, \quad i=1, \ldots, N-1, \\
y=y_{j}=j \Delta y, \quad-\infty \leq j \leq \infty,
\end{gathered}
$$

$$
\Phi(-1, y, t)=0 \text {. }
$$


Then

$$
\left\|\Phi_{x}(x, y, t)\right\| \leq\left\|\Phi_{x}(x, y, 0)\right\|
$$

where

$$
\|f\|^{2}=\int_{-\infty}^{\infty} \int_{-1}^{1} \frac{(1-x) f^{2}}{\sqrt{1-x^{2}}} d x d y .
$$

Proof. By taking the Fourier transform of (6.20) with respect to $y$ we find

$$
\begin{gathered}
\frac{\partial \phi_{x}(x, \xi, t)}{\partial t}=-4 \frac{\sin ^{2} \xi \Delta y}{(\Delta y)^{2}} \phi(x, \xi, t), \\
\text { at } x=x_{i}, \quad i=1, \ldots, N-1, \\
\phi(-1, \xi, t)=0,
\end{gathered}
$$

where $\phi(x, \xi, t)$ is the Fourier transform of $\Phi(x, y, t)$.

Multiplying $(6.23)$ by $\omega_{i}\left(1-x_{i}\right) \bar{\phi}_{x}\left(x_{i}, \xi, t\right)$ and summing over $i=1, \ldots, N-1$, where the $\omega_{i}$ are defined in (5.4), we obtain

$$
\frac{\partial}{\partial t} \sum_{i=0}^{N}\left(1-x_{i}\right) \omega_{i}\left|\phi_{x}\left(x_{i}, \xi, t\right)\right|^{2}=-4 \frac{\sin ^{2} \xi \Delta y}{(\Delta y)^{2}} \sum_{i=0}^{N}\left(1-x_{i}\right) \omega_{i}\left|\phi\left(x_{i}, \xi, t\right)\right|_{x}^{2}
$$

Since $(1-x)|\phi(x, \xi, t)|_{x}^{2}$ is a polynomial of degree $2 N-2$ in $x$, we may use the Gauss integration formula to obtain

$$
\frac{\partial}{\partial t} \sum_{i=0}^{N}\left(1-x_{i}\right) \omega_{i}\left|\phi_{x}(x, \xi, t)\right|^{2}=-4 \frac{\sin ^{2} \xi \Delta y}{(\Delta y)^{2}} \int_{-1}^{1}(1-x) \frac{|\phi|_{x}^{2}}{\sqrt{1-x^{2}}} d x .
$$

Integrating by parts and applying the Parseval equality, one obtains the desired result (6.22).

7. The Two-Dimensional Heat Equation. The same technique for investigating the eigenvalues of an operator may be implemented for other two-dimensional equations, for example the heat equation:

$$
\begin{gathered}
u_{t}=u_{x x}+u_{y y}, \\
u( \pm 1, y, t)=0, \\
\frac{\partial u}{\partial y}(x, \pm 1, t)=0, \\
u(x, y, 0)=u_{0}(x, y) .
\end{gathered}
$$

Conditions (7.2) may be replaced by $u_{x}( \pm 1, y, t)=0$, or more generally by

$$
\begin{gathered}
\alpha_{1} u(1, y, t)+\beta_{1} u_{x}(1, y, t)=0, \\
\gamma_{1} u(-1, y, t)+\delta_{1} u_{x}(-1, y, t)=0,
\end{gathered}
$$

as long as $\alpha_{1}, \beta_{1}, \gamma_{1}, \delta_{1}$ satisfy the conditions of the theorem proved in [9], i.e.,
(a) $\alpha_{1}, \beta_{1}, \gamma_{1}>0$ and $\delta_{1}<0$, or
(b) $\gamma_{1} \geq 0, \delta_{1} \leq 0$, or
(c) $\alpha_{1}=0$ or $\beta_{1}=0$.

One may also replace (7.3) by more general conditions in a similar way. 
THEOREM 7.1. The eigenvalues of the problem

$$
\begin{aligned}
& \lambda \Phi_{N M}(x, y, \lambda)=D_{N}^{(1)}(x) \Phi_{N M}(x, y, \lambda)+D_{M}^{(1)}(y) \Phi_{N M}(x, y, \lambda), \\
& \text { for } x=x_{i}=\cos \frac{\pi i}{N}, \quad i=1, \ldots, N-1 \text {, } \\
& y=y_{j}=\cos \frac{\pi j}{M}, \quad j=1, \ldots, M-1, \\
& \Phi_{N M}( \pm 1, y, \lambda)=0 \text {, } \\
& \frac{\partial}{\partial y} \Phi_{N M}(x, \pm 1, \lambda)=0
\end{aligned}
$$

are real and negative.

Proof. Expand $\Phi_{N M}(x, y, \lambda)$ as a sum of eigenfunctions of (6.5)-(6.6):

$$
\phi_{N M}(x, y, \lambda)=\sum_{k=1}^{M-1} a_{k}(x, \lambda) \Psi_{k}(y) .
$$

Substituting (7.8) into (7.5) yields

$$
\begin{array}{rlrl}
\lambda \sum_{k=1}^{M-1} a_{k}(x, \lambda) \Psi_{k}(y) & =\sum_{k=1}^{M-1} a_{k}^{\prime \prime}(x, \lambda) \Psi_{k}(y)+\sum_{k=1}^{M-1} \mu_{k} a_{k}(x) \Psi_{k}(y), \\
\text { for } \quad x=x_{i} & =\cos \frac{\pi i}{N}, & 1 \leq i \leq N-1, \\
y=y_{j} & =\cos \frac{\pi j}{M}, & 1 \leq j \leq M-1,
\end{array}
$$

where $\mu_{k}$ are the eigenvalues of $(6.5)-(6.6)$, which are real, distinct and negative by [9]. Since

$$
\left(\begin{array}{c}
\Psi_{k}\left(y_{1}\right) \\
\vdots \\
\Psi_{k}\left(y_{M-1}\right)
\end{array}\right)_{k=1, \ldots, M-1}
$$

are linearly independent, we have

$$
\lambda a_{k}(x, \lambda)=a_{k}^{\prime \prime}(x, \lambda)+\mu_{k} a_{k}(x, \lambda) \quad \text { at } x=x_{i}, 1 \leq i \leq N-1,
$$

or

$$
\begin{aligned}
\left(\lambda-\mu_{k}\right) a_{k}(x, \lambda)-a_{k}^{\prime \prime}(x, \lambda) & =\left(C_{k}+D_{k} x\right) T_{N}^{\prime}(x), \\
a_{k}( \pm 1, \lambda) & =0,
\end{aligned}
$$

where (7.10) is a consequence of (7.6).

Using the results in [9] and looking at the eigenvalue problem

$$
\begin{gathered}
\nu a_{k}(x, \lambda)-a_{k}^{\prime \prime}(x, \lambda)=\left(C_{k}+D_{k} x\right) T_{N}^{\prime}(x), \\
a_{k}( \pm 1, \lambda)=0,
\end{gathered}
$$

we conclude that the values of $\nu$ are real and negative and that there are $N-1$ linearly independent eigenfunctions corresponding to (7.11)-(7.12). Hence,

$$
\lambda-\mu_{k}=\nu_{l},
$$


or

$$
\lambda_{k, l}=\mu_{k}+\nu_{l}<0 .
$$

There are $(N-1)(M-1)$ eigenvalues and the same number of linearly independent eigenfunctions of (7.5)-(7.7), and it is easy to see that the eigenfunctions are products of the one-dimensional eigenfunctions, i.e.,

$$
\Phi_{N M}^{(k, l)}(x, y)=\Psi_{k}(y) \Psi_{l}(x),
$$

where $\Psi_{k}(y)$ is an eigenfunction of $(6.5)-(6.6)$ and $\Phi_{l}(x)$ is an eigenfunction of (7.11)-(7.12).

For the two-dimensional heat equation, we may prove also stability of the Chebyshev pseudospectral approximation.

THEOREM 7.2. Consider the semidiscrete pseudospectral approximation $\Phi_{N M}$ for the two-dimensional heat equation, satisfying

$$
\begin{array}{rlrl}
\frac{\partial}{\partial t} \Phi_{N M}(x, y, t) & =D_{N}^{(1)}(x) \Phi_{N M}(x, y, t)+D_{M}^{(1)}(y) \Phi_{N M}(x, y, t), \\
\text { at } \quad x & =x_{i}=\cos \frac{\pi i}{N}, & 1 \leq i \leq N-1, \\
y & =y_{j}=\cos \frac{\pi j}{N}, & 1 \leq j \leq M-1,
\end{array}
$$

$$
\begin{aligned}
& \Phi_{N M}( \pm 1, y, t)=0 \\
& \Phi_{N M}(x, \pm 1, t)=0
\end{aligned}
$$

where

$$
D_{N}^{(1)}(x)=P_{N M} \frac{\partial^{2}}{\partial x^{2}} P_{N M}, \quad D_{M}^{(1)}(y)=P_{N M} \frac{\partial^{2}}{\partial y^{2}} P_{N M}
$$

and

$$
P_{N M} u(x, y)=\sum_{k=0}^{N} \sum_{l=0}^{M} a_{k l} T_{k}(x) T_{l}(y)
$$

$a_{k l}$ being chosen such that

$$
P_{N M} u\left(x_{i}, y_{j}\right)=u\left(x_{i}, y_{j}\right), \quad 0 \leq i \leq N, 0 \leq j \leq M
$$

Then

$$
\left\|\Phi_{N M}(x, y, t)\right\|_{N M} \leq\left\|\Phi_{N M}(x, y, 0)\right\|_{N M}
$$

where

$$
\|\phi\|_{N M}^{2}=\frac{\pi^{2}}{N M} \sum_{i=0}^{N} \sum_{j=0}^{M} \phi^{2}\left(x_{i}, y_{j}, t\right)
$$

Proof. We multiply (7.13) by $\Phi_{N M}\left(x_{i}, y_{j}, t\right)$ and sum over $1 \leq i \leq N-1$, $1 \leq j \leq M-1$. Since (7.14)-(7.15) is valid, we may include the indexes $i=0, N$, 
$j=0, M$ in the summation:

$$
\begin{aligned}
\frac{1}{2} \frac{\partial}{\partial t} \frac{\pi^{2}}{N M} \sum_{i=0}^{N} \sum_{j=0}^{M} \Phi_{N M}^{2}\left(x_{i}, y_{j}, t\right) \\
=\frac{\pi^{2}}{N M} \sum_{j=0}^{M} \sum_{i=0}^{N} \frac{\partial^{2}}{\partial x^{2}} \Phi_{N M}\left(x_{i}, y_{j}, t\right) \Phi_{N M}\left(x_{i}, y_{j}\right) \\
\quad+\frac{\pi^{2}}{N M} \sum_{i=0}^{N} \sum_{j=0}^{M} \frac{\partial^{2}}{\partial y^{2}} \Phi_{N M}\left(x_{i}, y_{j}, t\right) \Phi_{N M}\left(x_{i}, y_{j}, t\right) .
\end{aligned}
$$

$\left(\left(\partial^{2} / \partial x^{2}\right) \Phi_{N M}\right) \Phi_{N M}$ is a polynomial of degree $2 N-2$ in $x,\left(\left(\partial^{2} / \partial y^{2}\right) \Phi_{N M}\right) \Phi_{N M}$ is a polynomial of degree $2 M-2$ in $y$, so by using the Gauss integration formula we have

$$
\begin{aligned}
\frac{\partial}{\partial t}\left\|\Phi_{N M}\right\|_{N M}^{2}= & \frac{\pi}{M} \sum_{j=0}^{M} \int_{-1}^{1} \frac{\left(\partial^{2} / \partial x^{2}\right) \Phi_{N M}(x, y, t) \Phi_{N M}(x, y, t)}{\sqrt{1-x^{2}}} d x \\
& +\frac{\pi}{N} \sum_{i=0}^{N} \int_{-1}^{1} \frac{\left(\partial^{2} / \partial y^{2}\right) \Phi_{N M}(x, y, t) \Phi_{N M}(x, y, t)}{\sqrt{1-y^{2}}} d y
\end{aligned}
$$

By $[10$, p. 82$]$ the right-hand side of $(7.16)$ is nonpositive, so that

$$
\frac{\partial}{\partial t}\left\|\Phi_{N M}\right\|_{N M}^{2} \leq 0
$$

Integration with respect to $t$ completes the proof.

8. Conclusions. We have shown that one may apply spectral methods to the small disturbance equation of transonic flow. The two schemes presented for solving this equation numerically are spectral in $x$ and either spectral in $y$ as well, or second-order in $y$.

The spatial operators of these schemes are proved to have eigenvalues with nonpositive real parts. Moreover, stability is proved for one of them. Similar statements can be proved for other two-dimensional problems, such as the two-dimensional heat equation.

Acknowledgment. I would like to thank my thesis advisor Professor David Gottlieb for his constructive suggestions.

This work is part of my Ph.D. thesis, and was supported in part at the Lawrence Berkeley Laboratory by the Applied Mathematical Sciences Subprogram of the Office of Energy Research, U.S. Department of Energy, under contract DE-AC0376SF00098.

\footnotetext{
Department of Mathematics and Lawrence Berkeley Laboratory

University of California

Berkeley, California 94720

E-mail:dalia@lbl-csam.arpa
}

1. W. F. Ballhaus \& P. M. Goorjian, Implicit Finite Difference Computation of Unsteady Transonic Flow about Airfoils, Including the Treatment of Irregular Shock Wave Methods, AIAA paper no. 77-205, (1977).

2. J. D. COLE, "Modern developments in Transonic flow," SIAM J. Appl. Math., v. 29, 1975, pp. 763-787. 
3. J. D. Cole \& A. F. MEssiter, "Expansion procedures and similarity laws for transonic flow, Part I: Slender bodies at zero incidence," Z. Angew. Math. Phys., v. 8, 1959, pp. 1-25.

4. B. ENGQUIST \& S. OSHER, "Stable and entropy satisfying approximations for transonic flow calculations," Math. of Comp., v. 34, 1980, pp. 45-75.

5. D. Fishelov, Application of Spectral Methods to Time Dependent Problems with Application to Transonic Flows, Ph.D. Thesis, Tel Aviv University, 1985.

6. D. FISHELOV, "Spectral methods for the small disturbance equation of transonic flows," SIAM J. Sci. Statist. Comput., v. 9, 1988, pp. 232-251.

7. D. GotTlieb, "The stability of pseudospectral Chebyshev methods," Math. Comp., v. 36, 1981, pp. 107-118.

8. D. Gotтlieb, "Strang-type difference schemes for multidimensional problems," SIAM J. Numer. Anal., v. 9, 1972, pp. 650-661.

9. D. GotTlieb \& L. Lustman, "The spectrum of Chebyshev collocation operator for the heat equation," SIAM J. Numer. Anal., v. 20, 1983, pp. 909-921.

10. D. Got tlieb \& S. ORSZAG, Numerical Analysis of Spectral Methods: Theory and Applications, CBMS Regional Conference Series in Applied Mathematics, SIAM, Philadelphia, Pa., 1977.

11. D. GotTlieb \& E. TURKEL, private communications, 1983.

12. A. JAMESON, "Numerical solution of nonlinear partial differential equations of mixed type," in: Numerical Solution of Partial Differential Equations III, Academic Press, New York, 1976, pp. 275-307.

13. E. Murman \& J. Cole, "Calculations of plane steady transonic flows," $A I A A J$., v. 9, 1971, pp. 114-121.

14. T. N. Phillipps, T. A. ZANG \& M. Y. Haussaini, "Preconditioners for the spectral multigrid method," IMA J. Numer. Anal., v. 6, 1986, pp. 273-292.

15. A. Solomonoff \& E. TURKel, Global Collocation Methods for Approximation and the Solution of Partial Differential Equations, ICASE Report No. 86-60, 1986.

16. H. TAL-EzER, "A pseudospectral Legendre method for hyperbolic equations with an improved stability condition," J. Comput. Phys., v. 67, 1986, pp. 145-172. 